\title{
L'éducation artistique dans le monde
}

Une étude internationale

Art education in the world: an international survey

La educación artística en el mundo. Un estudio internacional

\section{Anne Bamford}

Traducteur : Yves Goujon

\section{(2) OpenEdition}

\section{Journals}

Édition électronique

URL : https://journals.openedition.org/ries/1107

DOI : 10.4000/ries. 1107

ISSN : 2261-4265

Éditeur

France Education international

Édition imprimée

Date de publication : 1 septembre 2006

Pagination : 119-130

ISSN : 1254-4590

\section{Référence électronique}

Anne Bamford, "L'éducation artistique dans le monde », Revue internationale d'éducation de Sèvres [En ligne], 42 I septembre 2006, mis en ligne le 14 novembre 2011, consulté le 05 juillet 2021. URL : http:// journals.openedition.org/ries/1107; DOI : https://doi.org/10.4000/ries.1107 


\section{L'éducation artistique dans le monde *}

\section{Une étude internationale}

\section{Anne Bamford}

En 2004-2005, l'UNESCO, en collaboration avec l'Australia Council for the Arts (The Council) et l'IFACCA (International Federation of Arts Councils and Culture Agencies) a commandé une étude ${ }^{1}$ pour déterminer l'impact (si impact il y a) des programmes artistiques sur l'éducation des enfants et des jeunes dans le monde. L'objectif était de déterminer la ligne de base du statut de l'éducation artistique dans le monde. Les résultats de l'étude montrent que l'art contribue grandement à l'éducation des enfants et qu'il a une incidence sur les résultats scolaires, le bien-être, l'attitude vis-à-vis de l'école et la perception de l'apprentissage. Cependant, ce qui caractérise l'éducation artistique varie considérablement d'un pays à l'autre.

Cet article analyse la portée et l'ampleur des pratiques et des politiques d'éducation artistique dans le monde et souligne l'importance de la qualité en termes d'apport global à l'éducation artistique. En particulier, l'analyse sera centrée sur la spécificité de l'enseignement artistique, sur son organisation et sur les conditions de mise en œuvre qui déterminent sa qualité.

\section{DES DÉFINITIONS ET DES OBJECTIFS MULTIPLES}

Prendre un cliché "instantané » de la situation mondiale de l'éducation artistique à un moment donné permet aux enseignants et aux responsables politiques d'accéder à une connaissance empirique des cadres d'organisation et des facteurs qui régulent et structurent l'enseignement artistique.

Pour commencer, il est important de reconnaître les faiblesses de cette approche. Toute recherche dans le domaine artistique est difficile. La conduite de cette étude a présenté bien évidemment des défis méthodologiques. D’un côté, il était nécessaire d'utiliser une définition relativement précise de l'art afin de rassembler des informations générales sur l'étendue, le contenu et l'impact des différents programmes. D’un autre côté, ces définitions se sont souvent avérées trop étroites pour représenter intégralement l'étendue de ces programmes.

Selon le sens commun, l'éducation artistique peut-être définie comme l'ensemble des activités qui visent à transmettre un héritage culturel aux jeunes

\footnotetext{
* Article traduit par Yves Goujon.

1. Bamford, 2006.
} 
et à leur permettre de comprendre et de créer leur propre langage artistique. Le contenu de l'enseignement artistique est varié et lié à un contexte spécifique au sein de chaque pays; cependant, il comporte des éléments communs à presque tous les pays. Ces composantes communes incluent la musique, le dessin, la peinture et l'artisanat, bien que la nature de ce dernier puisse différer. Les médias nouveaux et émergents, comme le cinéma, la photographie, l'étude des médias, la conception et l'art assistés par ordinateur font régulièrement partie de l'éducation artistique dans les pays développés.

Dans les pays en développement, les définitions de l'art sont larges et témoignent d'une conscience culturelle et d'une connaissance solide des traditions et des manières de vivre. Par exemple, à la Barbade, les danses et chants folkloriques (landship, stilt-walking, tukbands) font partie du programme d'éducation artistique, tandis qu'au Sénégal, l'art floral, l'art des nombres, le batik, la céramique, le conte oral, la mode, la coiffure, la fabrication d'accessoires vestimentaires et la couture font tous partie du programme d'éducation artistique. Le terme namibien Ngoma indique également que l'acte artistique a une portée ou une fonction plus large que l'art lui-même. L'art prépare l'individu et la communauté au travail ; que ce travail soit simple ou plus profond, il apporte un éclairage spirituel et éducatif. Le terme Ngoma indique que l'art est part intégrante de la société.

Il existe une tendance à formuler des définitions plus strictes de l'éducation artistique qui admettent mal la multiplicité des significations et des pratiques. Par exemple, on n'imagine pas une conférence internationale ayant pour thème la couture, la fabrication des tresses, le Ngoma ou les tuk bands, et cetera. Cette tendance à des définitions plus globales de l'éducation artistique a aussi conduit à une hiérarchie implicite, même si elle n'était pas intentionnelle, appliquée à l'éducation artistique. Le résultat de cette compétition signifie que, parmi les arts dans le monde, les arts visuels et la musique tendent à avoir la primauté tandis que le théâtre, la danse et d'autres formes artistiques reçoivent très peu d'attention de la part des autorités éducatives.

La situation générale de l'enseignement artistique dans le monde semble avoir peu changé au cours des années : les objectifs culturels, sociaux et esthétiques continuent d'être la principale justification avancée pour l'existence d'une éducation artistique. Parallèlement, comme les professeurs d'art gagnent en reconnaissance en Asie, il existe une volonté d'examiner plus en détail les objectifs spirituels inhérents à l'éducation artistique. Par exemple, en Inde, on note un désir de reconnaitre les bénéfices pour le corps et l'esprit de formes artistiques comme la méditation et le yoga. De même, en Malaisie et au Bhoutan, on accorde une grande attention à l'harmonie née de la pratique des arts. On mène des recherches sur les bénéfices éducatifs de l'art : il contribue au bonheur de l'enfant et à son épanouissement total. Ce lien de l'éducation artistique à des principes de base ésotériques est aussi présent dans les pays 
d'Amérique du Sud et dans les Caraïbes où ces principes soulignent non seulement les bénéfices thérapeutiques et intérieurs, mais admettent la capacité de l'éducation artistique à construire la citoyenneté et à donner un sens d'appartenance à une communauté et à une démocratie. Dans plusieurs pays musulmans d'Afrique et du Moyen-Orient, il existe un lien puissant entre la vie religieuse du pays et la contribution de certains genres artistiques à la construction et au maintien du développement spirituel.

Depuis le milieu des années 1950, les textes officiels sur l'éducation artistique insistent sur la créativité artistique des enfants. Mais ce n'est pas, dans la plupart des pays, la seule raison pour inclure les arts dans l'éducation. L'utilité de l'art pour la construction individuelle et l'identité culturelle de l'enfant est plus importante encore.

\section{Patrimoine culturel et éducation artistique}

Il existe un grand débat sur la place de l'éducation culturelle dans l'éducation artistique. En Europe du Nord, la notion de "canons » culturels a une influence directe sur l'éducation artistique. L'art y est explicitement envisagé comme un moyen d'influencer le développement culturel de l'enfant. Il existe un débat mondial pour savoir si l'élément culturel - souvent transmis par l'éducation artistique - relève de la diversité culturelle ou s'il s'agit de préserver un patrimoine culturel. La question est de savoir si ces canons culturels peuvent admettre la diversité culturelle et s'ils contribuent à promouvoir une participation et un engagement artistique plus grands.

L'étude du patrimoine culturel est le noyau dur des programmes artistiques dans de nombreux pays, même si le rôle de l'éducation artistique dans la construction de la culture est moins clair: joue-t-elle une part active dans le développement culturel, ou transmet-elle simplement la culture d'une histoire culturelle dominante? On observe des liens étroits entre les arts et l'éducation culturelle dans les pays européens et ces liens semblent plus apparents dans les États relativement récents fondés au cours des cent à cent cinquante dernières années. Les chercheurs ont montré que le processus de construction nationale dans les pays neufs était souvent lié à un effort conscient d'établir une culture « haut de gamme»(Gellner 1981).

En marge de ce débat culturel, ce lien étroit entre patrimoine culturel et art peut conduire indirectement à un élargissement des définitions et du contenu de l'enseignement artistique. Par exemple, au Danemark, on se demande si la couture, la cuisine et le travail du bois doivent être intégrés à l'éducation artistique. De manière similaire, on peut argumenter que le sport devrait côtoyer l'art sous la bannière de l'éducation culturelle et le débat pourrait s'étendre parce que de nombreux secteurs d'activités culturelles pourraient arguer de leurs mérites éducatifs et de leur relation à l'identité individuelle et 
collective d'une nation. Aux Fidji, par exemple, le sport dépend de l'éducation artistique car le lien entre le mouvement et la pratique artistique est si fort que la place du sport en tant qu'enseignement culturel est implicite. Mais cela pose des problèmes aux enseignants d'art fidjiens qui craignent que le sport ne prenne l'avantage sur les autres pratiques artistiques créatrices.

La présence ou l'absence de la littérature et de l'alphabétisation au sein des programmes artistiques semble diverger selon les pays. Plusieurs pays incluent les ateliers d'écriture et de littérature dans les arts au sein des politiques nationales, mais ces activités ne sont pas nécessairement enseignées sous le label "éducation artistique ». Alors que l'on considère que la poésie et la fiction font largement parties de l'héritage culturel dans différents pays, leur lien avec l'art est très variable. En Irlande, par exemple, on considère que la poésie et la pratique orale du conte sont sans aucun doute un art et forment une part indispensable de l'éducation artistique d'un enfant. De même, dans beaucoup de pays africains et du Pacifique, les traditions orales que sont le chant et le conte forment le noyau dur des expériences artistiques des jeunes enfants.

Dans le contexte mondial, l'éducation artistique est très étroitement liée à la pratique d'une activité artistique. Il ne fait pas de doute que, au niveau mondial et au niveau local, il existe des savoirs ancestraux qui ne peuvent être acquis que par une pratique artistique. Un seul pays dans l'étude a mentionné le rôle particulier joué par les musées et la critique d'art dans les programmes, mais on peut présumer que cette idée est le cadre structurel qui englobe la peinture, le dessin, la musique, et cetera.

De même, l'esthétique en tant que sujet spécifique n'est mentionnée que par la République slovaque, mais on peut supposer qu'elle existe aussi dans d'autres dimensions des programmes d'enseignement artistique.

\section{LA PLACE de L’ÉdUCATION ARTISTIQUE DANS LES POLITIQUES ÉDUCATIVES}

Malgré des définitions variables et des différences de contenu selon les pays, les arts, sous une forme ou une autre, font partie des politiques éducatives de presque tous les pays du monde. Même si l'on peut arguer que la seule présence de l'éducation artistique dans la politique éducative n'est pas la garantie de moyens appropriés ou de qualité dans les classes, il est significatif que, au niveau des gouvernements au moins, il soit reconnu que l'art contribue à l'éducation de l'enfant. Même dans des pays ou des États où la place de l'éducation artistique n'est pas respectée au sein de l'enseignement général, il existe au moins la reconnaissance tacite qu'il est important pour les enfants d'avoir une expérience artistique et d'être engagés dans la vie culturelle d'un pays. Cependant, il y a toujours une différence considérable entre ce qui est obligatoire dans un pays et la nature et la qualité du programme d'enseignement artistique que les enfants reçoivent 
réellement dans les écoles. Il semble qu'il y ait un abîme entre l'opinion générale selon laquelle l'art est bon pour les enfants, et le laissez-faire en ce qui concerne le contrôle de la qualité de l'éducation artistique reçue par les enfants.

\section{Des textes officiels aux pratiques}

L'éducation artistique est obligatoire à l'école dans $84 \%$ des pays. Dans ces pays, $94 \%$ des personnes interrogées ont déclaré que l'éducation artistique était enseignée comme une discipline indépendante. En moyenne, 176 heures annuelles dans les écoles primaires et 165 heures annuelles dans les écoles secondaires sont consacrées à l'enseignement artistique ${ }^{2}$. Plusieurs pays indiquent des variations importantes en ce qui concerne le temps consacré à cet enseignement artistique. Par exemple, aux États-Unis, il existe des « différences délirantes selon les écoles et les communautés, avec des écoles qui ne bénéficient pratiquement pas d'éducation artistique quelle qu'elle soit et des écoles où, là où elle existe, les cours durent quarante minutes ". Le National Center for Education Statistics of the U.S. Department of Education (centre national pour les statistiques en éducation du ministère de l'éducation) a découvert que, au cours de l'année 1999-2000, l'enseignement artistique avait été de quarante-six heures annuelles dans l'enseignement public primaire et de quarante-quatre heures dans le secondaire.

Des personnes interrogées lors de l'étude internationale ont aussi pointé une variation entre les exigences "officielles» et ce qui se passe en pratique. Cela semble être le cas dans plusieurs pays indépendamment de leur économie. Par exemple, en Australie, l'enseignement artistique est obligatoire dans tous les systèmes et on lui alloue généralement un temps spécifique dans les programmes du secondaire. Mais dans les écoles primaires, la mise en œuvre de ce temps réservé dépend grandement des écoles, ainsi que de l'intérêt pour l'art et de la qualité du professeur.

Alors que l'éducation artistique fait partie de la politique éducative dans pratiquement tous les pays, on note une différence considérable entre ce qui est obligatoire et la nature et la qualité des programmes d'éducation artistique reçus en réalité par les enfants dans les écoles. Les données indiquent que, dans $55 \%$ des pays, la réussite de certains programmes artistiques a eu un impact sur les stratégies des politiques nationales d'éducation artistique. Cependant, dans $46 \%$ des pays, malgré des exemples de réussite de programmes artistiques en cours, on a observé peu ou pas de modifications conséquentes de l'éducation artistique ou de la politique éducative en général. Il semble donc que la situation suivante soit générale : alors que la plupart des gouvernements prétendent favoriser la promotion de la culture par une politique en faveur de

2. Énoncé dépourvu de sens en anglais, il doit manquer un mot. 
l'éducation artistique, il existe un fossé entre la politique annoncée en matière d'enseignements artistiques et la réalité des moyens médiocres dans les classes.

En général, les politiques éducatives dans le monde ignorent superbement l'art ou le considèrent comme un enseignement marginal. Dans $59 \%$ des cas, la politique éducative générale ne reconnaît pas du tout la contribution de l'éducation artistique. Il est évident que même si l'éducation artistique existe sur «le papier » dans de nombreux pays, elle n'est ni valorisée ni reconnue au sein des programmes, et n'a pas d'existence pratique dans la classe. Soit la politique générale d'éducation ignore l'éducation artistique, soit l'éducation artistique est marginalisée par des pratiques qui ne conduisent pas à des programmes de qualité. La curiosité, la pensée «décalée », l'apprentissage à partir d'un projet sont limités par des méthodes pédagogiques unidimensionnelles, des classes surchargées, des stocks de matériel de base médiocres et inégaux et un accès limité ou inexistant des élèves aux nouvelles technologies de l'information.

\section{Un risque de marginalisation}

Cet exemple souligne la complexité de la place de l'éducation artistique au sein de l'enseignement général, et ce particulièrement en période de restriction. Jusque dans les pays économiquement développés, il est difficile de trouver une place pour l'art dans l'agenda éducatif. Pourtant, même dans les pays bénéficiant d'excellents programmes d'éducation artistique, tels que ceux qui sont mis en œuvre en Finlande, les impératifs économiques amènent à réduire la place des arts dans la politique générale d'éducation, comme le suggère cette phrase relevée en Finlande : «aujourd'hui, les écoles ont moins d'argent et de ressources, et consacrent moins de leçons à l'art qu'il y a quelques années» (Bamford 2006).

L'appréciation de la valeur de l'art dans la politique générale d'éducation d'un pays semble être un cas classique de ce qu'on appelle la "variabilité selon le parcours " (path-dependency), dans laquelle des expériences menées au niveau local (généralement des écoles) engendrent une compréhension de la valeur d'une éducation artistique poussée. Cependant, ces expériences ne sont pas communiquées comme elles le devraient, ni appréciées par les décideurs au sommet de la hiérarchie éducative. Ce point est mis en valeur dans la réponse faite par l'Autriche qui suggère " qu'il y a peut-être eu une influence sur la politique éducative dans des situations scolaires individuelles, mais pas de manière structurelle».

\section{Le rôle des institutions culturelles et du supérieur}

Il faut également souligner le rôle de la politique d'enseignement supérieur dans le succès des politiques d'éducation artistique. Par exemple, à la 
Barbade, la création de cours de création artistique dans les universités fut une avancée positive vers une politique d'éducation artistique plus large et vers le développement de filières éducatives réelles dans le domaine artistique.

Des exemples en Colombie montrent aussi l'importance du soutien des institutions culturelles pour la construction d'une reconnaissance de la valeur de l'enseignement artistique au sein de la politique générale d'éducation. Dans le cas présent, les efforts combinés d'un certain nombre de bureaux d'étude ont amélioré le statut de l'éducation artistique au sein de la politique générale d'éducation.

Un mouvement récent d'artistes, de professeurs, de décideurs gouvernementaux et d'experts en éducation a développé des stratégies pour valoriser l'importance de l'éducation culturelle et artistique dans les apprentissages. On peut citer à titre d'exemples le Proyecto RED de la Universidad Nacional et le Proyecto de Investigación del IDEP de la Secretaría del Distrito Capital. Le ministre de la culture a récemment conduit un séminaire sur les politiques d'éducation artistique et une équipe permanente de recherche a été créée pour prolonger le travail entamé pendant le séminaire. L'objectif principal de cette équipe de recherche est de concevoir une politique publique pour ce champ éducatif en prenant comme point de départ l'articulation entre éducation artistique formelle et non formelle.

Il est également significatif de remarquer que, alors que le gouvernement et l'enseignement supérieur jouent un rôle clé et décident si l'éducation artistique peut être implantée avec succès dans un pays, un certain nombre d'institutions jouent un rôle essentiel et s'assurent du suivi de l'enseignement artistique. Des fondations, le secteur caritatif, des églises, des syndicats et des personnes privées jouent tous un rôle pivot en s'assurant que l'éducation artistique est dispensée dans les écoles et les institutions culturelles.

Compte tenu de la diversité des organisations impliquées, il est clair que le soutien de nombreuses institutions est nécessaire pour doter les écoles de programme d'éducation artistique. Il est par conséquent évident que si la mise en œuvre d'une politique artistique revient au gouvernement, elle nécessite dans le même temps l'appui d'organisations non gouvernementales (ONG) et d'institutions culturelles qui contribuent à réhausser la qualité de l'enseignement artistique dispensé.

\section{Le mécénat}

Dans de nombreux pays, on pense que les dons des fondations et des mécènes sont versés au profit des représentations sur scène ainsi qu'aux événements culturels et qu'il est difficile, pour l'éducation artistique, d'entrer dans la compétition pour obtenir ces dons. En particulier, dans de nombreux cas, les fonds publics provenant de secteurs autres que le secteur éducatif ou d'agences 
non gouvernementales sont utilisés pour les besoins de représentations ou d'expositions dans les écoles ou à l'extérieur, de manière exceptionnelle.

Par exemple, au Danemark, les dons consacrés à l'éducation artistique peuvent être utilisés pour subventionner des spectacles et des artistes dans des écoles ou des programmes éducatifs dans des institutions culturelles. Ces programmes peuvent mener directement ou non à une éducation artistique poussée ou garantir des expériences éducatives de qualité pour tous les enfants. En eux-mêmes, ces dons sont rarement considérés comme faisant partie d'un projet stratégique global pour l'éducation artistique et peuvent être symboliques, inefficaces, ou encore être modestement capitalisés dans le cadre d'une éducation de qualité. Il est souvent admis que les partenariats avec nombre d'ONG procurent plus que des ressources financières. Ce point est manifeste dans la partie de l'étude consacrée au Canada.

L'enseignement artistique peut bénéficier d'une grande variété de ressources pour des raisons très différentes, qu'elles soient purement financières, qu'elles donnent accès à des moyens ou favorisent le volontariat, ou grâce au mécénat. Les bénéfices attribués à l'éducation artistique résultent aussi d'une demande croissante d'une main-d'œuvre créative et de collectivités créatives désireuses d'attirer les entreprises.

\section{Politiques culturelles ET POLITIQUES ÉDUCATIVES}

Alors qu'il faudrait considérer les mérites distincts de l'éducation, de l'art et des organismes culturels pour construire des liens plus étroits, il existe souvent un partage problématique des budgets alloués à l'art et à la culture d'un côté, et à l'éducation de l'autre.

De plus en plus de politiques culturelles et artistiques reconnaissent l'utilité de s'investir auprès des jeunes et de se tenir informé de ce qui se passe dans l'éducation. Cependant, on note aussi des critiques de l'approche actuelle qui consiste à considérer l'éducation des enfants et des jeunes comme une façon de «construire le public de l'avenir». Cette approche place les intérêts des jeunes pour l'art et la culture dans une sorte de capsule temporelle et suggère qu'en "farcissant " l'esprit du jeune enfant d'une dose suffisante d'art et de culture, on peut espérer que dans sa vie future, cette dose latente d'art pourra être rentable en termes de clientèle et de public pour les arts. Cette approche est insultante pour l'enfant. Elle ne reconnaît pas que les enfants sont déjà des consommateurs avertis et enthousiastes de l'art et de la culture dès aujourd'hui, et pas seulement demain. De plus, nous savons que les décisions des enfants en matière d'art et de culture ont un effet profond sur eux en tant qu'individus ainsi que sur les décisions de leurs parents et de la société, plus généralement. Supposer qu'une décision culturelle est prise une fois qu'un individu atteint l'âge de dix-huit ans est un déni de la réalité sociale des jeunes. 
Dans cette mesure, l'éducation artistique ne devrait pas être de façon trop restrictive réservée aux enfants (les adultes ont certainement aussi besoin d'être éduqués à un large spectre artistique), et la politique culturelle ne devrait pas se limiter au rapport des adultes à l'art et à la culture.

Alors que l'on a admis pendant un temps que l'éducation artistique avait des effets bénéfiques sur l'enfant, on a moins d'informations sur la façon dont une éducation artistique efficace peut donner matière à une politique culturelle et artistique plus large au-delà de la salle de classe. L'influence de l'éducation artistique sur la politique culturelle semble émerger de liens plus étroits entre les arts et les autorités éducatives. Par exemple, au Canada, il existe une tendance, ces dernières années, à aller vers plus de collaboration entre les ministères de l'éducation et de la culture, entre les éducateurs et les artistes.

Inversement, une politique culturelle efficace peut conduire en réalité à une meilleure éducation artistique dans les écoles. C'est le cas en Allemagne où, par exemple, la politique artistique de la ville de Munich est influencée par les effets positifs des projets d'éducation artistique et culturelle pour les enfants et les jeunes.

L'étude montre également que les autorités culturelles et artistiques ont montré ces dernières années un intérêt plus grand pour la politique et les pratiques d'éducation artistique. Aux Pays-Bas, on note que " des changements positifs dans la politique artistique ont entraîné des projets d'éducation artistique » et que « les décideurs de la politique artistique ont montré un intérêt accru pour l'éducation artistique ». Alors que dans certains pays, les autorités artistiques et éducatives travaillent de façon plus concertée, dans beaucoup de cas, les dissensions entre les autorités artistiques et éducatives ont un impact contraire sur la mise en œuvre de la politique d'éducation artistique. Cette diversification dans la répartition des budgets ne présente pas qu'un intérêt théorique.

Il est inutile de dire que la mise en œuvre des politiques est d'une importance cruciale. Sans les programmes, la totalité de l'action politique est dénuée de sens. Cependant, on a prêté trop peu d'attention à cette question. Dans les domaines éducatifs et culturels, les acteurs politiques ont adopté, avec plus ou moins d'esprit critique, une approche hiérarchique de la mise en œuvre politique : ils sont partis du principe que les politiques développées par le gouvernement étaient mises en œuvre par des antennes (comme les écoles et autres organismes d'éducation artistique). Cette approche n'a pas produit les résultats escomptés.

\section{L'ENJEU DE L'ÉQUITÉ ET DE LA QUALITÉ}

La relation complémentaire, et quelquefois conflictuelle, entre les diffuseurs/fournisseurs d'art et de culture et le secteur éducatif revient souvent à un rapport aléatoire entre les moyens alloués au sein de l'école (grâce à une politique 
éducative officielle) et les moyens alloués à l'extérieur de l'école (considérés comme la part facultative d'une politique non officielle). Alors que des programmes non officiels et extrascolaires se révèlent de grande qualité et fructueux, la prudence est de rigueur quant à une éventuelle marginalisation de l'art dans le secteur non formel. Par exemple, alors que la Chine propose dans ses centres aérés et de loisirs d'excellents programmes artistiques, le programme officiel est mené par «l'économie » et "l'éducation artistique est toujours très marginalisée ». Cette division entre secteurs officiels et secteurs non officiels est exacerbée par des divisions entre les autorités chargées de l'éducation scolaire et celle qui ont la responsabilité de l'attribution des budgets. Cette complexité est éclairée par l'exemple finlandais, où le ministère de l'éducation se compose d'un département éducatif (d'où un ministre de l'éducation) et d'un département culturel (d'où un ministre de la culture). Le département culturel est très actif dans son soutien à l'éducation artistique extrascolaire, mais le gouvernement a réduit les cours d'art dans les nouveaux cœurs de programmes. On note un bon système d'éducation artistique en dehors de l'école, mais seulement $12 \%$ des enfants âgés de sept à dixhuit ans peuvent en bénéficier. Le ministère de l'éducation a attribué à toutes les formes artistiques un noyau de programme selon deux configurations, une générale et une approfondie. Le problème est donc que l'éducation artistique dépend de l'intérêt que lui portent les familles quand l'école obligatoire n'a plus les moyens d'offrir une bonne éducation artistique de base.

Cet exemple souligne les questions clés d'équité liées à la marginalisation de l'éducation artistique. Son existence n'est possible que dans les secteurs non officiels et n'est plus assurée par une politique officielle d'éducation et un programme. Ceci est illustré en Colombie où la politique éducative menée par le ministère de la culture «s'est principalement orientée vers une éducation artistique non formelle qui est devenue la principale stratégie de promotion de l'art ». Si des budgets d'origines diverses peuvent apporter beaucoup à l'éducation des enfants, ils ne doivent pas se substituer aux dotations officielles, systématiques et chiffrables dans les écoles. Ou alors, si une éducation artistique devenait le centre de toutes les attentions, il faudrait allouer des moyens suffisants pour s'assurer que tous les enfants reçoivent une éducation artistique substantielle.

Outre la controverse sur les dotations officielles et celles qui ne le sont pas et le besoin de partenariats entre les fournisseurs d'éducation et de culture, la question de la qualité est un facteur déterminant pour savoir quels bénéfices peuvent être retirés de l'éducation artistique.

À la différence de l'enseignement de la lecture, de l'écriture et du calcul, pour lesquels les enseignants ont reçu une formation spécifique, les enseignants généralistes sont généralement chargés de l'enseignement artistique, avec peu voire pas de formation à l'éducation artistique. On pourrait se demander si ce manque de formation spécifique est l'une des raisons pour lesquelles les artistes et la collectivité jouent un rôle croissant dans l'éducation artistique. 
Des partenariats avec des organismes culturels et des expériences hors du milieu scolaire font indéniablement partie intégrante d'une éducation artistique de qualité. Cependant, ces partenariats ne devraient pas remplacer des programmes de qualité dans les écoles. Il n'est pas toujours vrai non plus qu'un artiste est la personne la plus à même d'encourager les apprentissages artistiques. Il ne suffit pas d'être un bon danseur, peintre, musicien, etc., pour être un bon enseignant dans ces domaines. Quantité d'études internationales montrent en réalité le contraire. Elles suggèrent qu'un professeur qualifié et passionné qui travaille aux côtés d'un artiste lui aussi impliqué forment la meilleure combinaison possible pour l'éducation artistique (Bamford 2006). Un tel schéma nécessite un partenariat véritable entre les différents acteurs, plutôt que des mesures épisodiques de rapprochement pour lesquelles on demande soit aux éducateurs de s'attaquer au manque d'intérêt et aux attentes des jeunes, soit aux artistes de pallier la médiocre qualité de l'éducation artistique dans le cadre scolaire.

Au sein des secteurs culturel et éducatif, il existe aussi un problème de responsabilité et de mise en œuvre. Pour résumer, il semble qu'il y ait un manque d'échanges d'idées entre ceux qui sont chargés de planifier ce qui est prévu pour l'éducation artistique et ceux qui sont chargés de réaliser ces projets. Par exemple, alors que les écoles sont sur le terrain pour mettre en œuvre l'éducation artistique, les professeurs, les artistes et les enfants sont rarement consultés sur le développement d'une politique d'éducation artistique.

Souvent, la mise en œuvre dans les écoles ne peut même pas répondre aux attentes fixées par la politique éducative par manque d'espace et de ressources humaines adéquats. L'éducation artistique semble médiocrement aidée au sein du secteur artistique et plus largement du secteur culturel. Tant qu'il se montre extrêmement motivé, le personnel enseignant créatif peut compenser des programmes sous financés et obtenir des résultats de qualité. Ceci ne devrait pas cautionner la défaillance en matière de développement des ressources matérielles et humaines. Le manque d'argent, des ressources inadaptées, un temps insuffisant et des structures rigides sont des facteurs susceptibles de limiter le succès de programmes artistiques poussés.

Le manque de formations spécialisées offertes aux éducateurs ou aux membres des professions artistiques qui enseignent la culture et l'art est particulièrement inquiétant. Les professeurs reçoivent peu ou pas de formation spécifique à l'éducation artistique et la situation est similaire chez les artistes et dans la collectivité, alors qu'ils jouent un rôle croissant dans l'éducation artistique. C'est particulièrement le cas de l'éducation artistique des jeunes enfants qui souffre du manque de professeurs spécialisés. Les enseignants généralistes chargés de l'éducation artistique des jeunes enfants ne sont pratiquement pas formés à l'éducation artistique. Des recherches comparatives en éducation ont montré que le moyen le plus efficace de s'assurer de la mise en œuvre des 
politiques décidées au niveau national est de reconnaître l'action des personnels sur le terrain et de développer des structures qui leur permettent de mettre en œuvre des programmes de qualité (Elmore 1981). Ajouter simplement une tâche à une liste déjà croissante d'activités, sans support adapté, n'a que peu de chances de mener à une éducation artistique fructueuse.

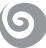

Pour conclure, l'étude commandée par l'UNESCO a montré que même si le combat pour inclure l'art dans une politique éducative a été largement couronné de succès, il n'a pas conduit à une mise en œuvre à grande échelle de programmes artistiques de qualité au niveau scolaire. Une éducation artistique de qualité permet d'augmenter la coopération, le respect, le sens de la responsabilité, la tolérance et l'esprit critique, et a un impact positif sur le développement de la compréhension scolaire, sociale et culturelle. De même, une éducation artistique médiocre ou une absence d'éducation artistique peut en réalité bloquer le développement de la créativité et de l'imagination. Le manque de temps, d'espace et de ressources est un facteur susceptible de limiter la réussite d'un programme artistique.

Le soutien politique aux arts n'a pas conduit à une large mise en ouvre de programmes de qualité à un niveau global. Le défi qui consiste à trouver des méthodes appropriées de recherche, d'évaluation et d'analyse signifie que la nécessité de faire des progrès dans l'approche de l'éducation artistique n'a pas toujours coulé de source. L'éducation artistique n'est pas le fruit d'une politique ou d'une commande mais plutôt d'un réseau complexe de forces sociales, de ressources appropriées et d'améliorations dans la mise en œuvre des politiques, y compris la formation professionnelle. Pour comprendre pleinement l'éducation artistique, il faut construire un lien entre les politiques et une éducation artistique de grande qualité.

\section{BibLIOGRAPHIE}

BAMFORD A. (2006): The Wow Factor: Global research compendium on the impact of the arts in education. Berlin, Waxmann Verlag.

GELLNER E. (1981) : Nations and nationalism. Oxford, Basil Blackwell.

The National Center for Education Statistics of the U.S. Department of Education (2000) : Survey of Arts Education within United States Schools. Washington. 